\title{
Antimicrobial Blue Light Inactivation of Polymicrobial Biofilms
}

\author{
Raquel Ferrer-Espada ${ }^{1,2}$, Xiaojing Liu', ${ }^{1,2}$ Xueping Sharon Goh ${ }^{1,2}$ and Tianhong Dai, ${ }^{1,2 *}$ \\ 1 Wellman Center for Photomedicine, Massachusetts General Hospital, Harvard Medical School, Boston, MA, United States, \\ ${ }^{2}$ Vaccine \& Immunotherapy Center, Massachusetts General Hospital, Harvard Medical School, Boston, MA, United States
}

OPEN ACCESS

Edited by:

Fabian Cieplik,

University Medical Center

Regensburg, Germany

Reviewed by:

Mariusz Stanislaw Grinholc, University of Gdańsk and Medical

University of Gdańsk, Poland

Andreas Pummer

University Medical Center

Regensburg, Germany

${ }^{*}$ Correspondence:

Tianhong Dai

tdai@mgh.harvard.edu

Specialty section:

This article was submitted to Antimicrobials, Resistance

and Chemotherapy,

a section of the journal

Frontiers in Microbiology

Received: 20 November 2018

Accepted: 21 March 2019

Published: 09 April 2019

Citation:

Ferrer-Espada R, Liu X, Goh XS and Dai T (2019) Antimicrobial Blue

Light Inactivation of Polymicrobial

Biofilms. Front. Microbiol. 10:721.

doi: 10.3389/fmicb.2019.00721
Polymicrobial biofilms, in which mixed microbial species are present, play a significant role in persistent infections. Furthermore, polymicrobial biofilms promote antibiotic resistance by allowing interspecies transfer of antibiotic resistance genes. In the present study, we investigated the effectiveness of antimicrobial blue light (aBL; $405 \mathrm{~nm}$ ), an innovative non-antibiotic approach, for the inactivation of polymicrobial biofilms. Dual-species biofilms with Pseudomonas aeruginosa and methicillin-resistant Staphylococcus aureus (MRSA) as well as with P. aeruginosa and Candida albicans were reproducibly grown in 96-well microtiter plates or in the CDC biofilm reactor for 24 or $48 \mathrm{~h}$. The effectiveness of aBL inactivation of polymicrobial biofilms was determined through colony forming assay and compared with that of monomicrobial biofilms of each species. aBL-induced morphological changes of biofilms were analyzed with confocal laser scanning microscopy (CLSM) and scanning electron microscopy (SEM). For 24-h old monomicrobial biofilms formed in 96-well microtiter plates, 6.30$\log _{10}$ CFU inactivation of $P$. aeruginosa, 2.33- $\log _{10}$ CFU inactivation of $C$. albicans and 3.48- $\log _{10}$ CFU inactivation of MRSA were observed after an aBL exposure of $500 \mathrm{~J} / \mathrm{cm}^{2}$. Under the same aBL exposure, 6.34- $\log _{10}$ CFU inactivation of $P$. aeruginosa and 3.11- $\log _{10}$ CFU inactivation of $C$. albicans were observed, respectively, in dualspecies biofilms. In addition, 2.37- and 3.40- $\log _{10}$ CFU inactivation were obtained in MRSA and $P$. aeruginosa, dual-species biofilms. The same $a B L$ treatment of the biofilms developed in the CDC-biofilm reactor for $48 \mathrm{~h}$ significantly decreased the viability of $P$. aeruginosa monomicrobial and polymicrobial biofilm when cocultured with MRSA (3.70- and 3.56- $\log _{10}$ CFU inactivation, respectively). 2.58- $\log _{10}$ CFU inactivation and 0.86- $\log _{10}$ CFU inactivation was detected in MRSA monomicrobial and polymicrobial biofilm when cocultured with $P$. aeruginosa. These findings were further supported by the CLSM and SEM experiments. Phototoxicity studies revealed a no statistically significant loss of viability in human keratinocytes after an exposure to $216 \mathrm{~J} / \mathrm{cm}^{2}$ and a statistically significant loss of viability after $500 \mathrm{~J} / \mathrm{cm}^{2}$. aBL is potentially an alternative treatment against polymicrobial biofilm-related infections. Future studies will aim to improve the efficacy of $\mathrm{aBL}$ and to investigate $\mathrm{aBL}$ treatment of polymicrobial biofilm-related infections in vivo.

Keywords: antimicrobial blue light, polymicrobial, biofilm, Pseudomonas aeruginosa, Staphylococcus aureus, Candida albicans, CDC biofilm reactor, endogenous photosensitizer 


\section{INTRODUCTION}

Microorganisms are prone to forming biofilms, surfaceassociated microbial communities that are extremely resistant to antimicrobials and the immune system (Costerton et al., 1999). Moreover, biofilm-forming microorganisms are far more resistant to antimicrobials than organisms in suspension (Dunne, 2002). It is reported that biofilms are involved in up to $65 \%$ of infections where they often lead to severe illness with a prolonged hospital stay, increased costs, and high mortality ( $\mathrm{La}$ Fuente-Núñez et al., 2014).

Most diseases have previously been characterized as monomicrobial in nature, likely due to the extensive use of culture-dependent isolation techniques. Now, with the advent of culture-independent analysis methodologies, several are becoming increasingly recognized as polymicrobial infections (Peters et al., 2012). Polymicrobial biofilms, in which mixed microbial species are present, are the dominant form of microbial life in nature (Harriott and Noverr, 2011; DeLeon et al., 2014). By including mixed microbial species in a single community, biofilms obtain numerous advantages, such as an enlarged gene pool with more efficient DNA sharing, quorum sensing systems, passive resistance, metabolic cooperation, and many other synergies (Wolcott et al., 2013). For instance, the gram-negative pathogen Pseudomonas aeruginosa is commonly found in mixed infections with the polymorphic fungus Candida albicans or the gram-positive bacterium Staphylococcus aureus (Harriott and Noverr, 2011; DeLeon et al., 2014). As such, P. aeruginosa and C. albicans are often found together in burn wound infections, contaminated catheters and chronic lung infections (Peleg et al., 2010). P. aeruginosa and S. aureus, on the other hand, have frequently been isolated from chronically infected wounds, chronic suppurative otitis media, indwelling medical devices, abnormal airways such as those in cystic fibrosis and other chronic obstructive lung diseases (Yadav et al., 2017).

$P$. aeruginosa can attach to the surface of C. albicans hyphae (but not yeast cells) and form dual-species biofilms (Peleg et al., 2010). Interspecies competition between $P$. aeruginosa and $C$. albicans enhances the production of virulence factors and increases mutability, and thus can alter the course of hostpathogen interactions in polymicrobial infections (Peleg et al., 2010; Trejo-Hernández et al., 2014). As a result, dual-species biofilms with $P$. aeruginosa and $C$. albicans play extensive roles in nosocomial infections and infection in immunocompromised individuals (Fourie et al., 2016).

S. aureus and $P$. aeruginosa are two versatile bacterial pathogens that are frequently found together in chronic wound infections (Stacy et al., 2016). Dual S. aureus and P. aeruginosa infections are more virulent and/or result in worse outcomes than the single infections caused by either species. The mutualistic and parasitic interactions drive the synergistic impact of the two species on the progression of infections (Nguyen and OglesbySherrouse, 2016).

Effective therapy for tackling the antimicrobial resistance in polymicrobial biofilms is lacking. There is a pressing need for the development of new strategies against polymicrobial biofilm infections. Antimicrobial blue light $(\mathrm{aBL})$ in the spectrum of
400 to $470 \mathrm{~nm}$, as an innovative light-based non-antibiotic strategy, has attracted increasing attention due to its intrinsic antimicrobial effect without the involvement of exogenous photosensitizers (Dai et al., 2012; Wang et al., 2017; Gwynne and Gallagher, 2018). The proposed mechanism of action of $\mathrm{aBL}$ involves the natural accumulation in microbial cells of photoactivable metal-free porphyrins such as uroporphyrin, coproporphyrin, and to a lesser extent protoporphyrin (Dai and Hamblin, 2017). These endogenous porphyrins absorb the Soret band of light (405-420 nm) and are subsequently excited to the triplet state, where singlet oxygen is generated (Dai et al., 2012; Dai and Hamblin, 2017; Wang et al., 2017; Gwynne and Gallagher, 2018). Singlet oxygen rapidly reacts with a wide range of cellular macromolecules and damages proteins, lipids, DNA, and RNA (Glaeser et al., 2011; Bumah et al., 2017). Additional mechanisms of action such as prophage activation have also been proposed (Yang et al., 2017). Similar to antimicrobial photodynamic therapy (Dai et al., 2009), aBL inactivation of microorganisms is thought to be a multi-target damaging process (Maisch, 2015). As a consequence, the likelihood for the development of aBL-resistance by microorganisms is less than that of antibiotic resistance.

In the present study, we investigated the effectiveness of aBL inactivation of polymicrobial biofilms in vitro. Monomicrobial and polymicrobial biofilms of $P$. aeruginosa, $S$. aureus and C. albicans were reproducibly grown in 96-well microtiter plates or the CDC biofilm reactor. The effectiveness of aBL inactivation of polymicrobial biofilms was determined through colony forming assay and compared with that of the monomicrobial biofilms of each species. Furthermore, the morphological changes of biofilms induced by aBL were analyzed with confocal scanning microscopy (CLSM) and scanning electron microscopy (SEM).

\section{MATERIALS AND METHODS}

\section{Blue Light Source}

For aBL irradiation, we used a light-emitting diode (LED) with peak emission at $405 \mathrm{~nm}$ and a full width at half maximum (FWHM) of $20 \mathrm{~nm}$ (M405L3, Thorlabs, United States). The irradiance on the surface of the target was measured using a PM100D power meter (Thorlabs). The distance between the LED aperture and the target was set at $3 \mathrm{~cm}$ for $92.6 \mathrm{~mW} / \mathrm{cm}^{2}$ and $4 \mathrm{~cm}$ for $60 \mathrm{~mW} / \mathrm{cm}^{2}$. The radiant exposure was calculated with the following equation (Radiant exposure $\left(\mathrm{J} / \mathrm{cm}^{2}\right)=$ Irradiance $\left(\mathrm{W} / \mathrm{cm}^{2}\right) \times$ Exposure time $\left.(\mathrm{s})\right)$.

\section{Microbial Strains and Culture Conditions}

The strains used in this study were $P$. aeruginosa IQ0046 (a clinical multidrug-resistant isolate) (Lu et al., 2018), C. albicans CEC 749 (Enjalbert et al., 2009), and MRSA USA300 (Vidlak and Kielian, 2016). These strains were routinely grown at $37^{\circ} \mathrm{C}$ and $150 \mathrm{rpm}$ in Brain Heart Infusion (BHI; BioMerieux, France) broth or BHI supplemented with $15 \mathrm{~g} / \mathrm{L}$ agar. 


\section{Biofilms in 96-Well Microtiter Plates Monomicrobial Biofilms}

C. albicans, MRSA and P. aeruginosa were grown overnight in BHI broth. Then, the cell densities of the suspensions were adjusted to approximately $10^{6} \mathrm{CFU} / \mathrm{mL}$ for MRSA and $P$. aeruginosa and $10^{5} \mathrm{CFU} / \mathrm{mL}$ for C. albicans, and $100 \mu \mathrm{L}$ aliquots were inoculated in 12 wells of 96-well microtiter plates (Wang et al., 2016). After 24 or 48 h of incubation (with renewal of the media every $24 \mathrm{~h}$ ), the biofilms were carefully washed two times with phosphate-buffered saline (PBS; Thermo Fisher Scientific, United States). Then, three wells of untreated biofilms were scraped and pooled together with a sterile pipette tip and this procedure was repeated twice with fresh PBS and in two technical replicates. The biofilms in the other 6 wells were irradiated with aBL at exposures of $216 \mathrm{~J} / \mathrm{cm}^{2}\left(60 \mathrm{~mW} / \mathrm{cm}^{2}, 60 \mathrm{~min}\right)$ or $500 \mathrm{~J} / \mathrm{cm}^{2}\left(92.6 \mathrm{~mW} / \mathrm{cm}^{2}\right.$, $90 \mathrm{~min}$ ) and the scraping procedure was repeated after $\mathrm{aBL}$ exposure. The total volume obtained, $600 \mu \mathrm{L}$, was sonicated for $5 \mathrm{~min}$ and the samples were diluted and processed for colony counting. The experiments were performed in triplicates for each condition.

\section{Dual-Species Biofilms}

The dual-species biofilms with $C$. albicans and $P$. aeruginosa were formed by mixing $10^{5} \mathrm{CFU} / \mathrm{mL}$ of $C$. albicans and $10^{6}$ $\mathrm{CFU} / \mathrm{mL}$ of $P$. aeruginosa in 12 wells of a 96-well microplate; and the dual-species biofilms with MRSA and P. aeruginosa were obtained by mixing $10^{6} \mathrm{CFU} / \mathrm{mL}$ of each species. After 24 or $48 \mathrm{~h}$ of incubation (with renewal of the media every $24 \mathrm{~h})$, the biofilms were exposed to $216 \mathrm{~J} / \mathrm{cm}^{2}\left(60 \mathrm{~mW} / \mathrm{cm}^{2}\right.$, $60 \mathrm{~min})$ or $500 \mathrm{~J} / \mathrm{cm}^{2}\left(92.6 \mathrm{~mW} / \mathrm{cm}^{2}, 90 \mathrm{~min}\right) \mathrm{aBL}$, collected and sonicated. To enumerate the CFUs, sonicated dual-species biofilms with $C$. albicans and $P$. aeruginosa were plated on BHI agar supplemented with chloramphenicol (Sigma-Aldrich, United States) for the selective growth of C. albicans or with amphotericin B for the selective growth of $P$. aeruginosa (Clinical and Laboratory Standards Institute et al., 2002, 2011). The sonicated dual-species biofilms with $P$. aeruginosa and MRSA were plated on BHI agar supplemented with colistin for selective growth of MRSA or vancomycin for selective growth of $P$. aeruginosa. Growth recovery on selective growth media was compared with growth recovery on a general medium (BHI without antibiotics). Three independent experiments were performed for each condition. To ensure thermal effect was not involved in killing microorganisms, the temperatures of the samples were monitored using a Traceable ${ }^{\circledR}$ Type K thermocouple (Thermo Fisher Scientific).

\section{Biofilms in the CDC Reactor}

Biofilms of $P$. aeruginosa, MRSA and C. albicans were also developed under dynamic shear conditions using the CDC biofilm reactor (model CBR 90-1 DH, BioSurface Technologies Corporation, United States) as described elsewhere (SánchezGómez et al., 2015; Fernández-Rivero et al., 2017). Briefly, $400 \mathrm{~mL}$ $\mathrm{BHI}(37 \mathrm{~g} / \mathrm{L})$ were inoculated at $37^{\circ} \mathrm{C}$ with $1 \mathrm{~mL}$ of an overnight culture of the microorganisms and incubated in the biofilm reactor with $150 \mathrm{rpm}$ stirring. The dual-species biofilms with
C. albicans and $P$. aeruginosa or with MRSA and $P$. aeruginosa were formed by inoculating the CDC biofilm reactor with $1 \mathrm{~mL}$ of each one of the strains. After $24 \mathrm{~h}$, a constant flow of $10 \mathrm{~mL} / \mathrm{min}$ BHI $(3.7 \mathrm{~g} / \mathrm{L})$ was stablished and dense biofilms $\left(10^{7}-10^{8} \mathrm{CFU} / \mathrm{cm}^{2}\right)$ were developed on the surface of small disks called coupons. After $24 \mathrm{~h}$ of further incubation under continuous flow (i.e., $48 \mathrm{~h}$ of incubation in total), coupons were removed from the chamber and planktonic cells were eliminated by rinsing with $1 \mathrm{~mL}$ sterile PBS. Then, coupons were immersed in $4 \mathrm{~mL}$ of sterile $\mathrm{PBS}$ in $3.5-\mathrm{cm}$ petri dishes and irradiated using aBL with exposures of $216\left(60 \mathrm{~mW} / \mathrm{cm}^{2}\right.$, $60 \mathrm{~min})$ or $500 \mathrm{~J} / \mathrm{cm}^{2}\left(92.6 \mathrm{~mW} / \mathrm{cm}^{2}, 90 \mathrm{~min}\right)$ and processed for colony counting.

For enumerating CFU, the biofilms were detached by scraping the coupon surface with a sterile wooden stick. Then, biofilm cells were suspended in $3 \mathrm{~mL}$ PBS and homogenized by $5 \mathrm{~min}$ of sonication (Bransonic 2510R-MT, United States) and vortexing (Thermo Fisher Scientific, United States). The aliquots were serially diluted, plated for CFU counting, and the cell densities in $\log _{10} \mathrm{CFU} / \mathrm{cm}^{2}$ of the biofilms attached to the coupons were calculated. In turn, the calculated cell densities in $\log _{10} \mathrm{CFU} / \mathrm{cm}^{2}$ allowed the determination of $\log _{10} \mathrm{CFU}$ inactivation, which was defined as the logarithm of the ratio of the $\mathrm{CFU} / \mathrm{cm}^{2}$ post-aBL exposures to the $\mathrm{CFU} / \mathrm{cm}^{2}$ of the untreated control. For the enumeration of $\mathrm{CFU}$ in the dual-species biofilm populations, sonicated samples were plated on BHI agar supplemented with chloramphenicol or amphotericin $\mathrm{B}$ for selective growth of C. albicans or P. aeruginosa; and on BHI agar supplemented with colistin or vancomycin for selective growth of MRSA or $P$. aeruginosa as previously described. Growth recovery on selective growth media was compared with growth recovery on a general medium (BHI without antibiotics). Experiments were independently repeated three times in duplicate coupons and the temperatures of the samples were also monitored as previously described.

\section{Visualization of Biofilms Using Confocal Laser Scanning Microscopy (CLSM)}

Biofilm structure and microbial viability in biofilms were also visualized using CLSM. In brief, aBL-treated $\left(500 \mathrm{~J} / \mathrm{cm}^{2}\right)$ and untreated monomicrobial and polymicrobial biofilms grown on the polycarbonate coupons of the CDC biofilm reactor were stained with SYTO 9 and propidium iodide (PI) (Invitrogen, United States). SYTO 9 is a dye that can diffuse through the membranes of microorganisms and stain DNA. On the other hand, PI can only penetrate the cells and dye DNA when the integrity of the membrane has been compromised. As a result, SYTO9 and PI work as an indicator of viability and death of microorganisms, respectively (Stiefel et al., 2015). However, PI can only be used for evaluating death of microorganisms when the membrane is damaged. In case bacteria are killed without membrane damage, death of bacteria may be understimated using this approach. After staining, the biofilms were examined with an Olympus Fluoview FV10i CLSM using the Alexa Fluor $488 \mathrm{~nm}$ and the $568 \mathrm{~nm}$ wavelength filters and the $10 \times$ or $60 \times$ objectives. 


\section{Visualization of Biofilms Using Scanning Electron Microscopy (SEM)}

Scanning electron microscopy was performed to investigate the morphological changes of biofilms induced by aBL treatment. Monomicrobial and polymicrobial biofilms were grown for $48 \mathrm{~h}$ in the CDC biofilm reactor and then exposed to $500 \mathrm{~J} / \mathrm{cm}^{2}$ of aBL $\left(92.6 \mathrm{~mW} / \mathrm{cm}^{2}, 90 \mathrm{~min}\right)$ or left untreated. After aBL exposure, the treated and untreated coupons were washed in PBS and fixed at $4^{\circ} \mathrm{C}$ for $24 \mathrm{~h}$ in $0.1 \mathrm{M}$ sodium cacodylate buffer containing $2.5 \%$ glutaraldehyde, $0.15 \%$ alcian blue, and $0.15 \%$ safranin $\mathrm{O}$. The fixed biofilms were washed three times for 10 min with 0.1 sodium cacodylate buffer, infiltrated with $1 \%$ osmium tetroxide for $1 \mathrm{~h}$, washed another three times for $10 \mathrm{~min}$ with 0.1 sodium cacodylate buffer and dehydrated from 30, 50, $70,85,95$, to $100 \%$ ethanol, spending $10 \mathrm{~min}$ in each solution and three consecutive exposures in the last concentration. Then, the samples were dried using a critical-point dryer, mounted on specimen stubs, sputter-coated with $8 \mathrm{~nm}$ platinum and examined using a high-resolution field emission SEM Hitachi S4800. Micrographs were acquired under high vacuum using an accelerating voltage of 2.0 or $3.0 \mathrm{kV}$.

\section{Examination of aBL Phototoxicity to Human Keratinocytes}

To test the phototoxicity of aBL to normal human epithelial cells, a monolayer of $\mathrm{HaCaT}$ keratinocytes were exposed to aBL at varying exposures of $216 \mathrm{~J} / \mathrm{cm}^{2}\left(60 \mathrm{~min}, 60 \mathrm{~mW} / \mathrm{cm}^{2}\right)$ and $500 \mathrm{~J} / \mathrm{cm}^{2}\left(90 \mathrm{~min}, 92.6 \mathrm{~mW} / \mathrm{cm}^{2}\right)$ in a $35 \mathrm{~mm}$ petri dish. The viability of the cells was measured using Cell Counting Kit-8 (CCK-8) (Sigma-Aldrich) after aBL exposure. CCK-8 uses WST-8 2-(2-methoxy-4-nitrophenyl)3-(4-nitrophenyl)-5-(2,4-disulfophenyl)-2H-tetrazolium, monosodium salt, which produces a water-soluble formazan dye upon bioreduction by cellular dehydrogenases. The absorbance was determined by the optical density at $450 \mathrm{~nm}$ (Molecular devices Emax).

\section{Statistical Analysis}

Data were presented as the mean \pm standard error. The differences between conditions were analyzed with a one-way ANOVA followed by Tukey's multiple comparison test (ns: not significant, $\left.{ }^{*} P<0.05,{ }^{* *} P<0.01,{ }^{* * *} P<0.001\right)$.

\section{RESULTS}

\section{aBL Inactivation of Monomicrobial and Polymicrobial Biofilms Grown in 96-Well Microtiter Plates 24-Hour Old Biofilms}

After $24 \mathrm{~h}$ of incubation, the obtained monomicrobial biofilms consisted of $7.76 \log _{10} \mathrm{CFU} / \mathrm{mL}$ of C. albicans, $8.62 \log _{10}$ $\mathrm{CFU} / \mathrm{mL}$ of MRSA and $8.87 \log _{10} \mathrm{CFU} / \mathrm{mL}$ of $P$. aeruginosa.

When C. albicans and P. aeruginosa were incubated together in dual-species biofilms for $24 \mathrm{~h}$, the cell densities decreased to $6.97 \log _{10} \mathrm{CFU} / \mathrm{mL}$ for C. albicans (ns, $P=0.159$ ) but increased to $9.42 \log _{10} \mathrm{CFU} / \mathrm{mL}$ for $P$. aeruginosa (ns, $P=0.534$ ). In the 24-hour old dual-species biofilms with MRSA and P. aeruginosa, the cell densities were 8.59 - and $8.16-\log _{10} \mathrm{CFU} / \mathrm{mL}$ for MRSA and $P$. aeruginosa, respectively (ns, $P>0.99$ and ns, $P=0.263$ ) (Table 1), in comparison to the cell densities in the respective monomicrobial biofilms.

The effectiveness of aBL inactivation of the biofilms varied depending on the species. In monomicrobial biofilms, $P$. aeruginosa was the most susceptible to aBL with $6.30 \log _{10}$ CFU reduction in 24-hour old biofilms after $500 \mathrm{~J} / \mathrm{cm}^{2} \mathrm{aBL}$ was delivered $(P<0.0001)$ (Table 1$)$. Under the same aBL exposure, 3.48- and 2.33- $\log _{10} \mathrm{CFU}$ reductions were observed in 24-hour old MRSA $(P<0.0001)$ and $C$. albicans biofilms $(P=0.0025)$, respectively.

In the 24-hour old dual-species biofilms with $P$. aeruginosa and C. albicans, 6.34- $\log _{10} \mathrm{CFU}$ reduction was observed in $P$. aeruginosa when an exposure of $500 \mathrm{~J} / \mathrm{cm}^{2}$ had been delivered. $(P<0.0001 ;$ Table 1), which was almost the same extent of inactivation as in the monomicrobial biofilms. However, C. albicans became more susceptible to aBL than in the monomicrobial biofilm and $3.11 \log _{10}$ CFU reduction was observed (Table 1; $P<0.0001$ ).

When the dual-species biofilms with $P$. aeruginosa and MRSA were exposed to $500 \mathrm{~J} / \mathrm{cm}^{2}$ aBL, $2.37 \log _{10} \mathrm{CFU}$ reduction was observed in MRSA $(P=0.0019)$ and $3.40 \log _{10}$ CFU reduction in $P$. aeruginosa $(P<0.0001)$ (Table 1$)$.

\section{8-Hour Old Biofilms}

After $48 \mathrm{~h}$ of incubation, the cell density in monomicrobial biofilms increased in all species in comparison to that of $24 \mathrm{~h}$ old biofilms (Table 2). In the dual-species biofilms with $P$. aeruginosa and $C$. albicans, the cell density of $P$. aeruginosa increased while the cell density of $C$. albicans decreased when the incubation time was increased from 24 to $48 \mathrm{~h}$ (Table 2). In the dual-species biofilms with $P$. aeruginosa and MRSA, the cell density of both species increased slightly (Table 2).

After treatment with $500 \mathrm{~J} / \mathrm{cm}^{2} \mathrm{aBL}$, the CFU reductions in the monomicrobial biofilms of each species were 2.11-, 2.35-, and 6.88- $\log _{10}$ for $C$. albicans $(P=0.0009)$, MRSA $(P<0.0001)$, and $P$. aeruginosa $(P<0.0001)$, respectively. For the dualspecies biofilms with C. albicans and P. aeruginosa, 3.41- and 7.41- $\log _{10}$ CFU reduction were observed after $500 \mathrm{~J} / \mathrm{cm}^{2}$ aBL exposure $(P<0.0001)$ (Table 2$)$, indicating that 1.30 - and 0.53 $\log _{10}$ CFU more inactivation of C. albicans and $P$. aeruginosa were achieved in the dual-species biofilms than in the monomicrobial ones.

On the other hand, 2.61- and 3.67- $\log _{10} \mathrm{CFU}$ reduction was obtained in MRSA $(P<0.0001)$ and $P$. aeruginosa $(P<0.0001)$, respectively, in the dual-species biofilms with the two species. In comparison to the monomicrobial biofilms of $P$. aeruginosa, aBLinduced reduction of $P$. aeruginosa decreased by $3.21-\log _{10} \mathrm{CFU}$ in the dual-species biofilms with MRSA and $P$. aeruginosa. In the case of MRSA, 0.26- $\log _{10}$ CFU more inactivation was observed in the polymicrobial biofilm with $P$. aeruginosa compared to the monomicrobial biofilm (Table 2).

The temperature was measured in the 96 -well microtiter plates during $405-\mathrm{aBL}$ exposure $\left(216\right.$ and $\left.500 \mathrm{~J} / \mathrm{cm}^{2}\right)$. The highest 
temperature recorded was $37.5^{\circ} \mathrm{C}$, at which no loss of the viability of any of the microorganisms was observed.

\section{aBL Inactivation of Biofilms Grown in the CDC Biofilm Reactor}

When the monomicrobial biofilms of each species were established in the CDC biofilm reactor under a turbulent flow of nutrients for $48 \mathrm{~h}$, the cell densities of the biofilms were $7.36 \log _{10}$ $\mathrm{CFU} / \mathrm{cm}^{2}$ for C. albicans, $9.23 \log _{10} \mathrm{CFU} / \mathrm{cm}^{2}$ for $P$. aeruginosa and $6.83 \log _{10} \mathrm{CFU} / \mathrm{cm}^{2}$ for MRSA (Table 3). When the dualspecies biofilms with $C$. albicans and $P$. aeruginosa were grown in the CDC biofilm reactor for the same time period, the cell densities changed to $5.83 \log _{10} \mathrm{CFU} / \mathrm{cm}^{2}$ for C. albicans and 8.07 $\log _{10} \mathrm{CFU} / \mathrm{cm}^{2}$ for P. aeruginosa (Table 3). For the 48 -h old dualspecies biofilms with MRSA and $P$. aeruginosa, the cell densities were 7.44 - and $7.52-\log _{10} \mathrm{CFU} / \mathrm{cm}^{2}$ for MRSA and $P$. aeruginosa, respectively (Table 3 ).

When an aBL exposure of $216 \mathrm{~J} / \mathrm{cm}^{2}$ had been delivered, 1.48and $2.05-\log _{10}$ CFU reduction were observed in $P$. aeruginosa and MRSA monomicrobial biofilms, respectively (ns, $P>0.05$ ), while only minimal reduction of CFU was observed in C. albicans monomicrobial biofilms. When the aBL exposure was increased to $500 \mathrm{~J} / \mathrm{cm}^{2}$, the aBL-induced inactivation of microorganisms in the respective monomicrobial biofilms were 1.04 - (ns, $P>0.05$ ),

TABLE 1 | Effectiveness of antimicrobial blue light $(405 \mathrm{~nm})$ inactivation of biofilms grown in 96 -well microtiter plates after $24 \mathrm{~h}$ of incubation.

\begin{tabular}{|c|c|c|c|c|c|c|c|c|c|c|}
\hline \multirow{2}{*}{$\begin{array}{l}\text { Biofilm type } \\
\text { Monomicrobial }\end{array}$} & \multicolumn{2}{|l|}{ Strain } & \multirow{2}{*}{$\begin{array}{c}\begin{array}{c}\text { Irradiance } \\
\left(\mathbf{m W} / \mathbf{c m}^{2}\right)\end{array} \\
60.0\end{array}$} & \multirow{2}{*}{$\begin{array}{c}\text { Irradiation } \\
\text { Time (min) } \\
60\end{array}$} & \multirow{2}{*}{$\begin{array}{c}\begin{array}{c}\text { Radiant } \\
\text { exposure }\left(\mathrm{J} / \mathrm{cm}^{2}\right)\end{array} \\
216\end{array}$} & \multirow{2}{*}{$\begin{array}{c}\begin{array}{c}\text { Biofilm cell density } \\
\log _{10}(\mathrm{CFU} / \mathrm{mL})\end{array} \\
7.58\end{array}$} & \multirow{2}{*}{$\begin{array}{c}\text { SE } \\
0.20\end{array}$} & \multicolumn{2}{|c|}{$\begin{array}{c}\text { Survival } \\
\text { bacterial counts } \\
\log _{10}(\mathrm{CFU} / \mathrm{mL})\end{array}$} & \multirow{2}{*}{$\frac{\text { SE }}{0.14}$} \\
\hline & C. albicans & CEC 749 & & & & & & -0.35 & ns & \\
\hline & & & 92.6 & 90 & 500 & 7.94 & 0.07 & -2.33 & $* *$ & 0.18 \\
\hline & MRSA & USA300 & 60.0 & 60 & 216 & 8.70 & 0.11 & -1.20 & ns & 0.20 \\
\hline & & & 92.6 & 90 & 500 & 8.54 & 0.27 & -3.48 & $* * *$ & 0.39 \\
\hline & $P$. aeruginosa & IQ0046 & 60.0 & 60 & 216 & 8.77 & 0.33 & -6.55 & $* * *$ & 0.45 \\
\hline & & & 92.6 & 90 & 500 & 8.97 & 0.86 & -6.30 & $* * *$ & 0.36 \\
\hline \multirow[t]{4}{*}{ Polymicrobial } & C. albicans & CEC 749 & 60.0 & 60 & 216 & 6.99 & 0.08 & -2.46 & $* *$ & 0.84 \\
\hline & & & 92.6 & 90 & 500 & 6.96 & 0.09 & -3.11 & $* * *$ & 0.43 \\
\hline & P. aeruginosa & IQ0046 & 60.0 & 60 & 216 & 9.98 & 0.17 & -5.67 & $* * *$ & 0.38 \\
\hline & & & 92.6 & 90 & 500 & 8.86 & 0.14 & -6.34 & $* * *$ & 0.20 \\
\hline \multirow[t]{4}{*}{ Polymicrobial } & MRSA & USA 300 & 60.0 & 60 & 216 & 8.23 & 0.09 & -1.42 & $\mathrm{~ns}$ & 0.24 \\
\hline & & & 92.6 & 90 & 500 & 8.96 & 0.14 & -2.37 & $* *$ & 0.23 \\
\hline & P. aeruginosa & IQ0046 & 60.0 & 60 & 216 & 8.05 & 0.08 & -3.94 & $* * *$ & 0.09 \\
\hline & & & 92.6 & 90 & 500 & 8.28 & 0.03 & -3.40 & $* * *$ & 0.34 \\
\hline
\end{tabular}

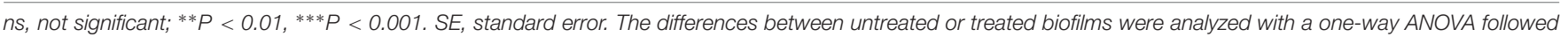
by Tukey's multiple comparison test.

TABLE 2 | Effectiveness of antimicrobial blue light (405 nm) inactivation of biofilms grown in 96-well microtiter plates after $48 \mathrm{~h}$ of incubation.

\begin{tabular}{|c|c|c|c|c|c|c|c|c|c|c|}
\hline \multirow{2}{*}{$\frac{\text { Biofilm type }}{\text { Monomicrobial }}$} & \multicolumn{2}{|l|}{ Strain } & \multirow{2}{*}{$\begin{array}{c}\begin{array}{c}\text { Irradiance } \\
\left.\text { (mW/cm }^{2}\right)\end{array} \\
60.0\end{array}$} & \multirow{2}{*}{$\begin{array}{c}\text { Irradiation } \\
\text { Time (min) }\end{array}$} & \multirow{2}{*}{$\begin{array}{c}\begin{array}{c}\text { Radiant } \\
\text { exposure }\left(\mathbf{J} / \mathbf{c m}^{2}\right)\end{array} \\
216\end{array}$} & \multirow{2}{*}{$\begin{array}{c}\begin{array}{c}\text { Biofilm cell density } \\
\log _{10}(\mathrm{CFU} / \mathrm{mL})\end{array} \\
8.41\end{array}$} & \multirow{2}{*}{$\begin{array}{c}\text { SE } \\
0.06\end{array}$} & \multicolumn{2}{|c|}{$\begin{array}{c}\text { Survival } \\
\text { bacterial counts } \\
\log _{10}(\mathrm{CFU} / \mathrm{mL})\end{array}$} & \multirow{2}{*}{$\frac{\text { SE }}{0.26}$} \\
\hline & C. albicans & CEC 749 & & & & & & -0.25 & ns & \\
\hline & & & 92.6 & 90 & 500 & 8.51 & 0.09 & -2.11 & $* * *$ & 0.09 \\
\hline & MRSA & USA300 & 60.0 & 60 & 216 & 8.77 & 0.13 & -1.62 & $*$ & 0.13 \\
\hline & & & 92.6 & 90 & 500 & 9.97 & 0.37 & -2.35 & $* * *$ & 0.23 \\
\hline & P. aeruginosa & IQ0046 & 60.0 & 60 & 216 & 9.34 & 0.23 & -3.67 & $* * *$ & 0.73 \\
\hline & & & 92.6 & 90 & 500 & 8.88 & 0.16 & -6.88 & $* * *$ & 0.16 \\
\hline \multirow[t]{4}{*}{ Polymicrobial } & C. albicans & CEC 749 & 60.0 & 60 & 216 & 6.84 & 0.30 & -2.96 & $* * *$ & 0.40 \\
\hline & & & 92.6 & 90 & 500 & 6.02 & 0.28 & -3.41 & $* * *$ & 0.21 \\
\hline & P. aeruginosa & IQ0046 & 60.0 & 60 & 216 & 9.45 & 0.18 & -5.48 & $* * *$ & 0.17 \\
\hline & & & 92.6 & 90 & 500 & 9.41 & 0.29 & -7.41 & $* * *$ & 0.29 \\
\hline \multirow[t]{4}{*}{ Polymicrobial } & MRSA & USA 300 & 60.0 & 60 & 216 & 8.50 & 0.24 & -2.44 & $* * *$ & 0.39 \\
\hline & & & 92.6 & 90 & 500 & 8.44 & 0.09 & -2.61 & $* * *$ & 0.08 \\
\hline & $P$. aeruginosa & IQ0046 & 60.0 & 60 & 216 & 8.80 & 0.12 & -4.19 & $* * *$ & 0.10 \\
\hline & & & 92.6 & 90 & 500 & 8.15 & 0.08 & -3.67 & $* * *$ & 0.24 \\
\hline
\end{tabular}

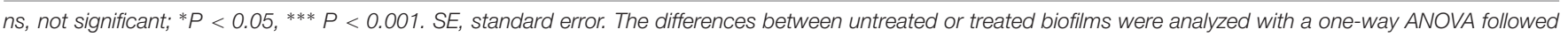
by Tukey's multiple comparison test. 
TABLE 3 | Antimicrobial blue light (405 nm) inactivation of biofilms grown in the CDC biofilm reactor after $48 \mathrm{~h}$ of incubation.

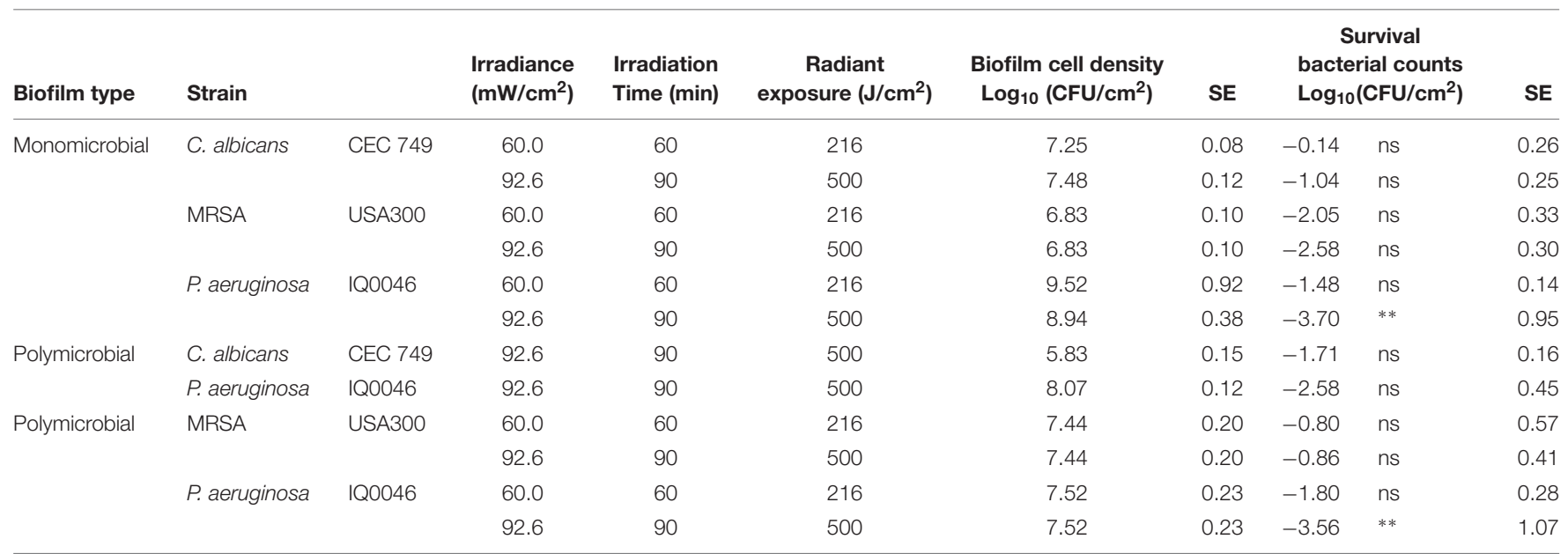

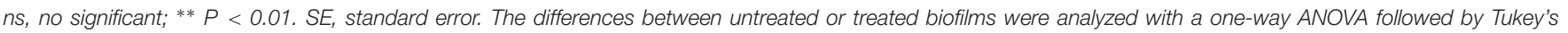
multiple comparison test.

2.58- (ns, $P>0.05)$ and $3.70-\log _{10} \mathrm{CFU}(P=0.0017)$ for

C. albicans, MRSA, and P. aeruginosa, respectively.

When the two species of $P$. aeruginosa and $C$. albicans were incubated together and formed dual-species biofilms, 2.58$\log _{10}$ CFU inactivation of $P$. aeruginosa and $1.71-\log _{10} \mathrm{CFU}$ inactivation of $C$. albicans were observed after treatment with $500 \mathrm{~J} / \mathrm{cm}^{2}$ aBL (ns, $P>0.05$ ). In the dual-species biofilms with MRSA and $P$. aeruginosa, aBL-induced reduction of CFU was 3.56- $\log _{10}$ and $0.86-\log _{10}$ for $P$. aeruginosa $(P=0.0029)$ and MRSA (ns, $P>0.05$ ), respectively.

Confocal laser scanning microscopy images confirmed that both monomicrobial and polymicrobial biofilms were successfully established in the CDC reactor (Figure 1). In all the untreated monomicrobial biofilms, spontaneously dead microorganisms (as stained red with PI) were observed. This event became more prevalent in the dual-species biofilms with $C$. albicans and $P$. aeruginosa, where some $C$. albicans hyphae also dyed with PI in the untreated biofilms. After an exposure of $500 \mathrm{~J} / \mathrm{cm}^{2} \mathrm{aBL}$, almost all the microbial cells in the biofilms appeared dead, as evidence by the red staining with PI in the CLSM images (Figure 1).

Scanning electron microscopy images revealed that aBL induced morphological changes in both the monomicrobial and polymicrobial biofilms (Figures 2, 3). Untreated C. albicans and $P$. aeruginosa monomicrobial biofilms showed highly dense and compact microbial cells (Figure 2). Untreated MRSA monomicrobial biofilm, on the other hand, had a smaller biofilm density and the surface of the polycarbonate coupon could be easily observed (Figure 3). All monomicrobial biofilms were surrounded with extracellular polymeric substance (EPS). $P$. aeruginosa and MRSA seemed to have a larger amount of EPS than C. albicans. The EPS of $P$. aeruginosa was organized like a mesh while the EPS of MRSA appeared in a star-like fashion. C. albicans biofilm was composed of yeast and hyphal forms.

After an aBL exposure of $500 \mathrm{~J} / \mathrm{cm}^{2}$ the density of the microbial cells in the monomicrobial biofilms decreased in all three species. The cell density decrease in $C$. albicans and MRSA monomicrobial biofilms was better observed with $\times 100$ or $\times 2.5 \mathrm{k}$ magnification. For $P$. aeruginosa monomicrobial biofilm, on the other hand, the decrease of cell density after $\mathrm{aBL}$ treatment was much more evident under $\times 25.0 \mathrm{k}$ magnification; although, they can also be observed in all the other magnifications.

In agreement with the CLSM images, $P$. aeruginosa attached to the surface of C. albicans in the dual-species biofilm and formed a very dense biofilm with lower amount of EPS than observed in the monomicrobial biofilms. The effect of the aBL treatment on the $P$. aeruginosa and $C$. albicans dual-species biofilm could be observed in the decrease in microbial cell density under $\times 100$ magnification for C. albicans and under $\times 25.0 \mathrm{k}$ magnification for $P$. aeruginosa (Figure 2 ).

The SEM images of the dual-species biofilm with MRSA and $P$. aeruginosa revealed that the two microorganisms are infiltrated together (Figure 3). Similar to the dual-species biofilm with $C$. albicans and P. aeruginosa, the cell density of the dualspecies biofilm with MRSA and $P$. aeruginosa decreased after treatment with $500 \mathrm{~J} / \mathrm{cm}^{2}$ aBL $\left(92.6 \mathrm{MW} / \mathrm{cm}^{2}\right.$ and $\left.90 \mathrm{~min}\right)$ ( $\times 2.5 \mathrm{k}$ magnification). In addition, $P$. aeruginosa and MRSA dual-species biofilm had lower amount and thinner EPS than the monomicrobial biofilms (Figure 3).

The temperature in the $35-\mathrm{mm}$ petri dishes was measured during $405-\mathrm{aBL}$ exposure $\left(216 \mathrm{~J} / \mathrm{cm}^{2}\right.$ and $\left.500 \mathrm{~J} / \mathrm{cm}^{2}\right)$. The highest temperature recorded was $33.1^{\circ} \mathrm{C}$.

\section{Phototoxicity of aBL to Normal Human Keratinocytes}

The phototoxicity study of aBL showed no statistically significant loss of viability in $\mathrm{HaCaT}$ cells after an exposure of up to $216 \mathrm{~J} / \mathrm{cm}^{2}\left(60 \mathrm{~mW} / \mathrm{cm}^{2}, 60 \mathrm{~min}\right)(P=0.4016)$ (Figure 4). The exposure of $\mathrm{HaCaT}$ cells to $500 \mathrm{~J} / \mathrm{cm}^{2}\left(92.6 \mathrm{~mW} / \mathrm{cm}^{2}, 90 \mathrm{~min}\right)$ reduced the metabolism of the cells significantly $(P=0.0011)$ to $49 \%$ of the unirradiated control (Figure 4). 


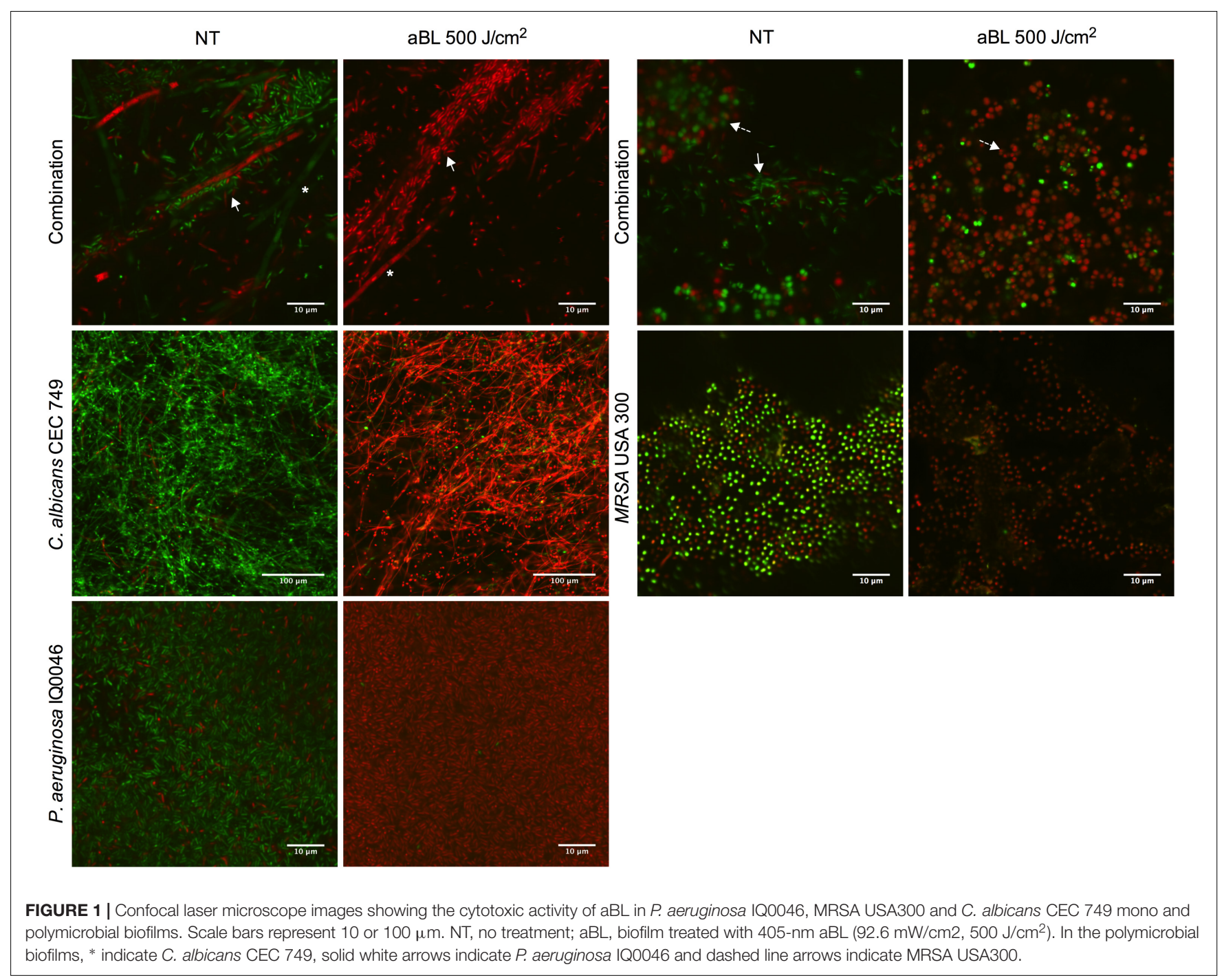

\section{DISCUSSION}

In this study, we investigated the efficacy of $405 \mathrm{~nm}$ aBL for the treatment of $P$. aeruginosa, MRSA, and C. albicans in monomicrobial and polymicrobial biofilms formed in 96well microtiter plates and the CDC biofilm reactor. Our findings demonstrated that in 96-well microtiter plates both the monomicrobial and polymicrobial biofilms (either 24 or $48 \mathrm{~h}$ old) were highly susceptible to $405 \mathrm{~nm}$ aBL. When the biofilms were developed in the CDC biofilm reactor, they exhibited higher tolerance to aBL. A possible reason for that result is the increased thickness of the biofilms obtained in the CDC biofilm reactor. Both techniques used in this study have their advantages and limitations. Microtiter plates are highthroughput and inexpensive but they suffer an exhaustion of nutrients and loosely attached biofilm can be detached during washing steps. The CDC biofilm reactor, on the other hand, is low-throughput and requires special equipment, but in this case, biofilms are grown with continuous supply of fresh medium and under shear stress (Azeredo et al., 2017), which is more clinically relevant. Hydrodynamics have major impact on biofilm growth, and biofilms grown under higher concentrations of nutrients and higher shear stress will be more abundant, filamentous and have higher cell density and thicker and more complex EPS. Those grown under lower nutrient concentrations and lower shear stress will be more compact with a lower cell density of a single layer of microcolonies and have thinner EPS. In addition, an open biofilm structure in the CDC biofilm reactor will facilitate the diffusion of nutrients easier than in the compact structure (Molobela and Ilunga, 2011).

Under most of the conditions, the CFU reduction by aBL in the C. albicans and P. aeruginosa dual-species biofilms was higher than that in the respective monomicrobial biofilms. Conversely, the CFU reduction by aBL obtained in the dualspecies biofilms with MRSA and P. aeruginosa was less than that obtained in the respective monomicrobial biofilms. This seemed to be in relation with the different kind of interactions between these specific pathogens. The results from the present study showed that an antagonistic interaction between C. albicans and $P$. aeruginosa in the dual-species biofilms favored aBL 


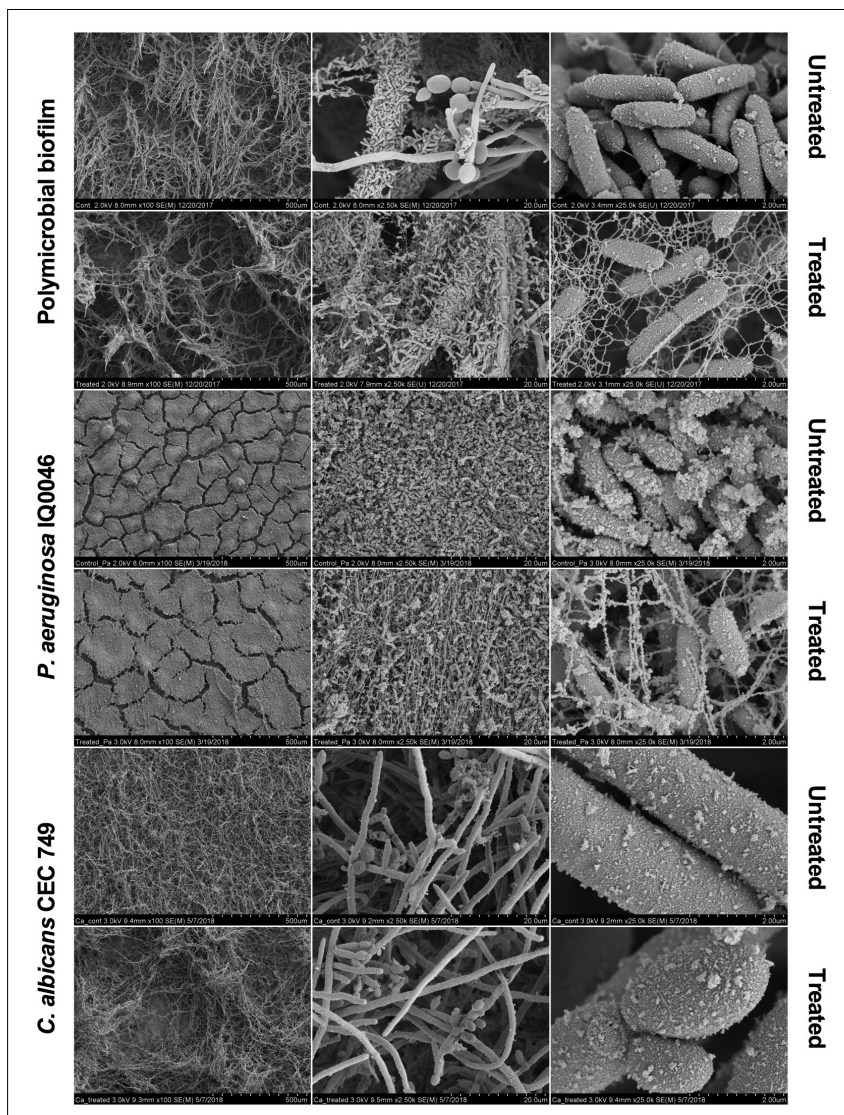

FIGURE 2 | Scanning electron microscope images showing the cytotoxic activity of aBL at $405 \mathrm{~nm}$ in P. aeruginosa IQ0046 and C. albicans CEC 749 monomicrobial and polymicrobial biofilms after an aBL exposure of $500 \mathrm{~J} / \mathrm{cm}^{2}$ (92.6 mW/cm² and $90 \mathrm{~min}$ ). Scale bars represent 500, 20, or $2 \mu \mathrm{m}$.

inactivation and a synergistic interaction between MRSA and $P$. aeruginosa appeared to hinder aBL inactivation.

To the best of our knowledge, this study is the first to investigate the activity of aBL at $405 \mathrm{~nm}$ against biofilms using the in vitro dynamic biofilm model CDC reactor and the effect of aBL at $405 \mathrm{~nm}$ against dual-species biofilms with $P$. aeruginosa and MRSA as well as with $P$. aeruginosa and $C$. albicans. Other polymicrobial biofilms where $\mathrm{aBL}$ has been tested include dualspecies biofilms formed by E. coli and S. aureus, in which 1.2 and $1.7 \log _{10} \mathrm{CFU} / \mathrm{mL}$ inactivation were obtained, respectively, after $254.66 \mathrm{~J} / \mathrm{cm}^{2}$ aBL at $405 \mathrm{~nm}$ was delivered (McKenzie et al., 2013) and in vivo human periodontal polymicrobial biofilms, in which an inactivation of 0.25 and $0.56 \log _{10} \mathrm{CFU} / \mathrm{mL}$ in Porphyromonas gingivalis and Prevotella intermedia was obtained, respectively, after $8.45 \mathrm{~J} / \mathrm{cm}^{2}$ aBL at $455 \mathrm{~nm}$ was delivered daily for 4 days (Soukos et al., 2015). In addition, a previous study showed that, after an exposure of $540 \mathrm{~J} / \mathrm{cm}^{2}$ at $415 \mathrm{~nm}$, no apoptotic cells developed in aBL-irradiated mouse skin (Wang et al., 2016).

The results presented in the CLSM (Figure 1) and SEM (Figures 2, 3) images as well as the cell densities of the monomicrobial and polymicrobial biofilms observed in the microtiter plates (Tables 1, 2) and in the CDC biofilm

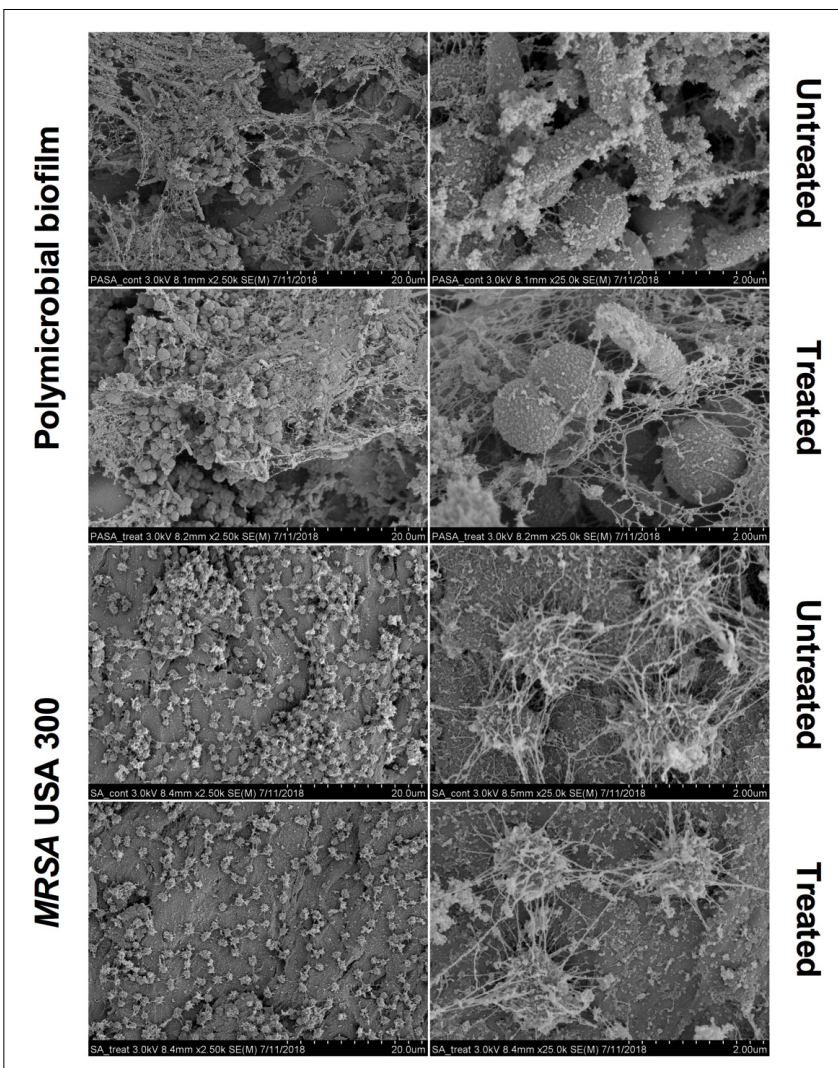

FIGURE 3 | Scanning electron microscope images showing the bactericidal activity of aBL at $405 \mathrm{~nm}$ in P. aeruginosa IQ0046 and MRSA USA300 monomicrobial and dual-species microbial biofilms after an aBL exposure of $500 \mathrm{~J} / \mathrm{cm}^{2}$ (92.6 mW/cm² and $90 \mathrm{~min}$ ). Scale bars represent 20 or $2 \mu \mathrm{m}$.

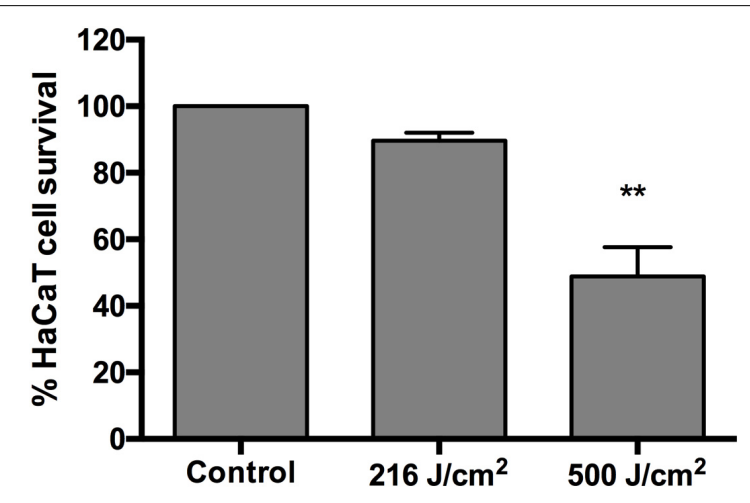

FIGURE 4 | Cytotoxicity of 405-nm aBL to normal human HaCaT cells after an exposure of $216 \mathrm{~J} / \mathrm{cm}^{2}\left(60 \mathrm{~mW} / \mathrm{cm}^{2}, 60 \mathrm{~min}\right)$ or $500 \mathrm{~J} / \mathrm{cm}^{2}$

(92.6 mW/cm², $90 \mathrm{~min}) .0$ vs $216 \mathrm{~J} / \mathrm{cm}^{2}(P=0.4016)$ and 0 vs $500 \mathrm{~J} / \mathrm{cm}^{2}$ $(P=0.0011)$. Bars are the SE. $* * P<0.01$.

reactor (Table 3) demonstrate the interactions between different microbial species in the polymicrobial biofilms. As described previously, $P$. aeruginosa formed its biofilm in the hyphae of C. albicans and as a result of that association, the hyphae of the fungus are damaged (Peleg et al., 2010). This resulted in 
reduced number of $C$. albicans $\mathrm{CFU}$ after $48 \mathrm{~h}$ of incubation with $P$. aeruginosa (Tables 2,3 ). In addition, the susceptibility of $C$. albicans to aBL increased in dual-species biofilms probably due to a synergistic effect with the damage to C. albicans cells induced by $P$. aeruginosa. On the other hand, the SEM images of the dual-species biofilms with MRSA and P. aeruginosa revealed that the two microorganisms are infiltrated together (Figure 3). The interaction between the two species seems to be synergistic as $P$. aeruginosa promotes MRSA adherence to the substrate in the turbulent environment of the CDC reactor. This is evidenced by the increase in MRSA cell density in the dual-species biofilms in comparison to the monomicrobial biofilm, and the decrease in EPS production observed in the SEM images (Table 3 and Figure 3). In addition, the tolerance of $P$. aeruginosa to $\mathrm{aBL}$ treatment increased when cocultured with MRSA. This can be attributed to the production of carotenoid pigments by MRSA, which are known to physically quench singlet oxygen and to protect bacteria from the lethal effects of aBL (Dahl et al., 1989; Glaeser et al., 2011).

As was reported in previous studies (Murdoch et al., 2013), aBL inactivation of microorganisms is oxygen dependent. Oxygen availability can be a concern in biofilms as actively respiring aerobic microcolonies can consume oxygen faster than oxygen diffusion, which results in the formation of anaerobic zones in the deep layers in the deep layers of the biofilm (Flemming et al., 2016). This explains the finding in the present study that the biofilms grown in the CDC biofilm reactor are more tolerant to aBL inactivation than the biofilms grown in the 96-well microplates. As the biofilms obtained in the CDC biofilm reactor are thicker than the biofilms grown in the 96well microplates, oxygen availability has a bigger impact on them. A potential way to increase the effectiveness of aBL inactivation of biofilms is to combine aBL with hyperbaric oxygen therapy. It was reported that hyperbaric oxygen therapy, in which the air pressure is several times higher than normal air pressure, could increase oxygen diffusion depth in biofilms and, subsequently, facilitate ciprofloxacin killing (Gade et al., 2018).

As shown in Tables 1, 2, an aBL exposure of $216 \mathrm{~J} / \mathrm{cm}^{2}$ reduced the viability of the polymicrobial biofilms very significantly. In

\section{REFERENCES}

Azeredo, J., Azevedo, N. F., Briandet, R., Cerca, N., Coenye, T., Costa, A. R., et al. (2017). Critical review on biofilm methods. Crit. Rev. Microbiol. 43, 313-351. doi: 10.1080/1040841X.2016.1208146

Bumah, V. V., Aboualizadeh, E., Masson-Meyers, D. S., Eells, J. T., Enwemeka, C. S., and Hirschmugl, C. J. (2017). Spectrally resolved infrared microscopy and chemometric tools to reveal the interaction between blue light $(470 \mathrm{~nm})$ and methicillin-resistant Staphylococcus aureus. J. Photochem. Photobiol. B Biol. 167, 150-157. doi: 10.1016/j.jphotobiol.2016.12.030

Clinical, and Laboratory Standards Institute (2002). Reference Method for Broth Dilution Antifungal Susceptibility Testing of Yeasts; Approved Standard, 2nd Edn. Wayne, PA: Clinical and Laboratory Standards Institute.

Clinical, and Laboratory Standards Institute (2011). Performance Standards for Antimicrobial Susceptibility Testing: Twenty-First Informational Supplement M100-S21. Wayne, PA: CLSI.

Costerton, J. W., Stewart, P. S., and Greenberg, E. P. (1999). Bacterial biofilms: a common cause of persistent infections. Science 284, 1318-1322. doi: 10.1126/ science. 284.5418 .1318 contrast, the phototoxicity study of aBL showed no statistically significant loss of viability in $\mathrm{HaCaT}$ cells after an exposure of up to $216 \mathrm{~J} / \mathrm{cm}^{2}$ (Figure 4). Significant cytotoxicity was observed in the HaCaT cells after an exposure of $500 \mathrm{~J} / \mathrm{cm}^{2} \mathrm{aBL}$.

The antimicrobial effect of aBL could be further improved synergizing its activity with a photosensitizer or antibiotic treatment. A synergistic combination could allow a decrease in the exposure time and radiant exposure, reducing the phototoxicity to the host cells and widening the therapeutic window of aBL.

The results obtained in the present study indicate the promising efficacy of aBL inactivation of polymicrobial biofilms, for treatment of infections in which multiple antimicrobials are usually required in order to eradicate different microbial species. Future studies are warranted to investigate other important biofilm-forming microbial species, the strategies to enhance the effectiveness of $\mathrm{aBL}$, and $\mathrm{aBL}$ treatment of polymicrobial biofilms-related infections in vivo.

\section{AUTHOR CONTRIBUTIONS}

RF-E, and TD designed the study. RF-E, XL, and XG performed the experiments. RF-E and TD analyzed the data and wrote the manuscript.

\section{FUNDING}

This study was supported in part by the National Institutes of Health (R01AI123312 to TD) and the United States Department of Defense (FA9550-16-1-0479 to TD).

\section{ACKNOWLEDGMENTS}

We would like to acknowledge William Fowle for his directions in preparing the SEM samples and Bin Zheng, Ph.D. for providing us the HaCaT cells.

Dahl, T. A., Midden, W. R., and Hartman, P. E. (1989). Comparison of killing of gram-negative and gram-positive bacteria by pure singlet oxygen. J. Bacteriol. 171, 2188-2194. doi: 10.1128/jb.171.4.2188-2194.1989

Dai, T., Gupta, A., Murray, C. K., Vrahas, M. S., Tegos, G. P., and Hamblin, M. R. (2012). Blue light for infectious diseases: Propionibacterium acnes, Helicobacter pylori, and beyond? Drug Resist. Updat. 15, 223-236. doi: 10.1016/j.drup.2012. 07.001

Dai, T., and Hamblin, M. R. (2017). Visible blue light is capable of inactivating Candida albicans and other fungal species. Photomed. Laser Surg. 35, 345-346. doi: $10.1089 /$ pho. 2017.4318

Dai, T., Huang, Y.-Y., and Hamblin, M. R. (2009). Photodynamic therapy for localized infections-state of the art. Photodiagnosis Photodyn. Ther. 6, 170-188. doi: 10.1016/j.pdpdt.2009.10.008

DeLeon, S., Clinton, A., Fowler, H., Everett, J., Horswill, A. R., and Rumbaugh, K. P. (2014). Synergistic interactions of Pseudomonas aeruginosa and Staphylococcus aureus in an in vitro wound model. Infect. Immun. 82, 4718-4728. doi: 10.1128/ IAI.02198-14

Dunne, W. M. (2002). Bacterial adhesion: seen any good biofilms lately? Clin. Microbiol. Rev. 15, 155-166. doi: 10.1128/CMR.15.2.155-166.2002 
Enjalbert, B., Rachini, A., Vediyappan, G., Pietrella, D., Spaccapelo, R., Vecchiarelli, A., et al. (2009). A multifunctional, synthetic Gaussia princeps luciferase reporter for live imaging of Candida albicans infections. Infect. Immun. 77, 4847-4858. doi: 10.1128/IAI.00223-09

Fernández-Rivero, M. E., Del Pozo, J. L., Valentín, A., de Diego, A. M., Pemán, J., and Cantón, E. (2017). Activity of amphotericin B and anidulafungin combined with rifampicin, clarithromycin, ethylenediaminetetraacetic acid, $\mathrm{N}$-acetylcysteine, and farnesol against Candida tropicalis biofilms. J Fungi 3:16. doi: 10.3390/jof3010016

Flemming, H.-C., Wingender, J., Szewzyk, U., Steinberg, P., Rice, S. A., and Kjelleberg, S. (2016). Biofilms: an emergent form of bacterial life. Nat. Rev. Microbiol. 14, 563-575. doi: 10.1038/nrmicro.2016.94

Fourie, R., Ells, R., Swart, C. W., Sebolai, O. M., Albertyn, J., and Pohl, C. H. (2016). Candida albicans and Pseudomonas aeruginosa interaction, with focus on the role of eicosanoids. Front. Physiol. 7:64. doi: 10.3389/fphys.2016.00064

Gade, P. A. V., Olsen, T. B., Jensen, P. Ø, Kolpen, M., Høiby, N., Henneberg, K. -Å., et al. (2018). Modelling of ciprofloxacin killing enhanced by hyperbaric oxygen treatment in Pseudomonas aeruginosa PAO1 biofilms. PLoS One 13:e0198909. doi: 10.1371/journal.pone.0198909

Glaeser, J., Nuss, A. M., Berghoff, B. A., and Klug, G. (2011). Singlet oxygen stress in microorganisms. Adv. Microb. Physiol. 58, 141-173. doi: 10.1016/B978-0-12381043-4.00004-0

Gwynne, P. J., and Gallagher, M. P. (2018). Light as a broad-spectrum antimicrobial. Front. Microbiol. 9:119. doi: 10.3389/fmicb.2018.00119

Harriott, M. M., and Noverr, M. C. (2011). Importance of Candida-bacterial polymicrobial biofilms in disease. Trends Microbiol. 19, 557-563. doi: 10.1016/ j.tim.2011.07.004

La Fuente-Núñez, C., Reffuveille, F., Haney, E. F., Straus, S. K., and Hancock, R. E. W. (2014). Broad-spectrum anti-biofilm peptide that targets a cellular stress response. PLoS Pathog. 10:e1004152. doi: 10.1371/journal.ppat.100 4152

Lu, M., Dai, T., Murray, C. K., and Wu, M. X. (2018). Bactericidal property of oregano oil against multidrug-resistant clinical isolates. Front. Microbiol. 9:2329. doi: $10.3389 /$ fmicb.2018.02329

Maisch, T. (2015). Resistance in antimicrobial photodynamic inactivation of bacteria. Photochem. Photobiol. Sci. 14, 1518-1526. doi: 10.1039/c5pp00037h

McKenzie, K., Maclean, M., Timoshkin, I. V., Endarko, E., MacGregor, S. J., and Anderson, J. G. (2013). Photoinactivation of bacteria attached to glass and acrylic surfaces by $405 \mathrm{~nm}$ light: potential application for biofilm decontamination. Photochem. Photobiol. 89, 927-935. doi: 10.1111/php.12077

Molobela, I. P., and Ilunga, F. M. (2011). Impact of bacterial biofilms: the importance of quantitative biofilm studies. Annl Microbiol. 62, 461-467. doi: 10.1007/s13213-011-0344-0

Murdoch, L. E., McKenzie, K., Maclean, M., MacGregor, S. J., and Anderson, J. G. (2013). Lethal effects of high-intensity violet 405-nm light on Saccharomyces cerevisiae, Candida albicans, and on dormant and germinating spores of Aspergillus niger. Fungal Biol. 117, 519-527. doi: 10.1016/j.funbio.2013.05.004

Nguyen, A. T., and Oglesby-Sherrouse, A. G. (2016). Interactions between Pseudomonas aeruginosa and Staphylococcus aureus during co-cultivations and polymicrobial infections. Appl. Microbiol. Biotechnol. 100, 6141-6148. doi: 10. 1007/s00253-016-7596-3

Peleg, A. Y., Hogan, D. A., and Mylonakis, E. (2010). Medically important bacterial-fungal interactions. Nat. Rev. Micro. 8, 340-349. doi: 10.1038/ nrmicro2313
Peters, B. M., Jabra-Rizk, M. A., O’May, G. A., Costerton, J. W., and Shirtliff, M. E. (2012). Polymicrobial interactions: impact on pathogenesis and human disease. Clin. Microbiol. Rev. 25, 193-213. doi: 10.1128/CMR.00013-11

Sánchez-Gómez, S., Ferrer-Espada, R., Stewart, P. S., Pitts, B., Lohner, K., and Martínez de Tejada, G. (2015). Antimicrobial activity of synthetic cationic peptides and lipopeptides derived from human lactoferricin against Pseudomonas aeruginosa planktonic cultures and biofilms. BMC Microbiol. 15:137. doi: 10.1186/s12866-015-0473-x

Soukos, N. S., Stultz, J., Abernethy, A. D., and Goodson, J. M. (2015). Phototargeting human periodontal pathogens in vivo. Lasers Med. Sci. 30, 943-952. doi: 10.1007/s10103-013-1497-9

Stacy, A., McNally, L., Darch, S. E., Brown, S. P., and Whiteley, M. (2016). The biogeography of polymicrobial infection. Nat. Rev. Microbiol. 14, 93-105. doi: 10.1038/nrmicro.2015.8

Stiefel, P., Schmidt-Emrich, S., Maniura-Weber, K., and Ren, Q. (2015). Critical aspects of using bacterial cell viability assays with the fluorophores SYTO9 and propidium iodide. BMC Microbiol. 15:36. doi: 10.1186/s12866-015-0376-x

Trejo-Hernández, A., Andrade-Domínguez, A., Hernández, M., and Encarnación, S. (2014). Interspecies competition triggers virulence and mutability in Candida albicans-Pseudomonas aeruginosa mixed biofilms. ISME J. 8, 1974-1988. doi: 10.1038/ismej.2014.53

Vidlak, D., and Kielian, T. (2016). Infectious dose dictates the host response during Staphylococcus aureus orthopedic-implant biofilm infection. Infect. Immun. 84, 1957-1965. doi: 10.1128/IAI.00117-16

Wang, Y., Wang, Y., Wang, Y., Murray, C. K., Hamblin, M. R., Hooper, D. C., et al. (2017). Antimicrobial blue light inactivation of pathogenic microbes: state of the art. Drug Resist. Updat. 3, 1-22. doi: 10.1016/j.drup.2017.10.002

Wang, Y., Wu, X., Chen, J., Amin, R., Lu, M., Bhayana, B., et al. (2016). Antimicrobial blue light inactivation of gram-negative pathogens in biofilms: in vitro and in vivo studies. J. Infect. Dis. 213, 1380-1387. doi: 10.1093/infdis/ jiw070

Wolcott, R., Costerton, J. W., Raoult, D., and Cutler, S. J. (2013). The polymicrobial nature of biofilm infection. Clin. Microbiol. Infect. 19, 107-112. doi: 10.1111/j. 1469-0691.2012.04001.x

Yadav, M. K., Chae, S.-W., Go, Y. Y., Im, G. J., and Song, J.-J. (2017). In vitro multi-species biofilms of methicillin-resistant Staphylococcus aureus and Pseudomonas aeruginosa and their host interaction during in vivo colonization of an otitis media rat model. Front. Cell Infect. Microbiol. 7:125. doi: 10.3389/fcimb.2017.00125

Yang, P., Wang, N., Wang, C., Yao, Y., Fu, X., Yu, W., et al. (2017). 460nm visible light irradiation eradicates MRSA via inducing prophage activation. J. Photochem. Photobiol. B Biol. 166, 311-322. doi: 10.1016/j.jphotobiol.2016. 12.001

Conflict of Interest Statement: The authors declare that the research was conducted in the absence of any commercial or financial relationships that could be construed as a potential conflict of interest.

Copyright () 2019 Ferrer-Espada, Liu, Goh and Dai. This is an open-access article distributed under the terms of the Creative Commons Attribution License (CC BY). The use, distribution or reproduction in other forums is permitted, provided the original author(s) and the copyright owner(s) are credited and that the original publication in this journal is cited, in accordance with accepted academic practice. No use, distribution or reproduction is permitted which does not comply with these terms. 